\title{
Past Experiences and Future Trends on Vertex Detector Cooling at LHC
}

\author{
Paolo Petagna $^{1}$ \\ CERN, PH Department \\ Route de Meyrin 385, Geneva, Switzerland \\ E-mail: paolo.petagna@cern.ch
}

Substantially different approaches have been adopted for the refrigeration plants of the first generation of vertex detectors at LHC: those of ALICE, ATLAS and CMS use PFC fluids, either in single phase or in a traditional Joule-Thomson cycle, while carbon dioxide in a pumped two-phase loop has been selected for the LHCb VELO. For what concerns the on-board thermal management of the sensors and related electronics, a traditional design has been followed, based on a common general approach and only differing in the specific choices related to the local configuration. Although the global performance of the detectors in this first phase of LHC operation can be claimed as fully satisfactory, it appears that the additional challenges posed by the coming upgrade phases can only be tackled through an effort on technology innovation and, in particular on much stronger and earlier integration of all the cooling-related aspects in the detector conception. Carbon dioxide seems to be the preferred choice for the refrigerant fluid of the future cooling plants (at least for cold operation), while advanced design approaches are pursued for the on-detector thermal management, integrating extended use of innovative materials and technologies. In this review paper, after a summary of the different approaches selected for the detectors presently in operation at LHC, the principal trends under development for the coming generations are discussed, for both off-detector and on-detector issues.

22nd International Workshop on Vertex Detectors (Vertex 2013)

September 15-20, 2013

Lake Starnberg, Germany

1

Speaker 


\section{Introduction}

The thermal management of silicon sensors and their related electronics in High Energy Physics (HEP) detectors has been historically considered an ancillary task, to be tackled when the design of the rest of the silicon detector is completed. This attitude might appear justified even for the complex Vertex detectors at LHC, in consideration of the relatively low surface power densities involved, typically of the order of $1 \mathrm{~W} / \mathrm{cm}^{2}$. On the other hand, e.g. in the field of high power computing chips, where surface power densities typically exceed $100 \mathrm{~W} / \mathrm{cm}^{2}$ and thermal management is often one of the main limiting factors, innovative cooling techniques are actually receiving growing attention in the last two decades. However, a simple order-ofmagnitude comparison between these two cases, like the one reported in Tab.1, also accounting for the total silicon surface and the heat dissipation volume typically involved, immediately shows that the volume power density is even higher for a Vertex detector, where the additional complication of the need for dozens of parallel cooling loops must also be managed. Further additional requirements are also specific to the HEP case: the minimization of material budget and space consumption; the lifetime design in excess of 10 years with extremely reduced maintenance possibilities; and, in most cases, a target temperature $<0{ }^{\circ} \mathrm{C}$. These considerations suggest that an efficient cooling should be thoroughly integrated in the stages of conception of future Vertex detectors. This includes both the "off-detector" issues linked to the cooling plant design, and the "on-detector" ones, related to the local thermal management of the detector modules. A review of the past experiences and future trends at LHC is proposed in this paper.

\begin{tabular}{|l|r|r|}
\hline & LHC Vertex detector & \multicolumn{1}{c|}{$\begin{array}{c}\text { High power } \\
\text { computing chips }\end{array}$} \\
\hline Surface Power Density & $\boldsymbol{O}\left(10^{0}\right) \mathrm{W} / \mathrm{cm}^{2}$ & $\boldsymbol{O}\left(10^{2}\right) \mathrm{W} / \mathrm{cm}^{2}$ \\
\hline Silicon Surface & $\boldsymbol{O}\left(10^{0}\right) \mathrm{m}^{2}$ & $\boldsymbol{O}\left(10^{-4}\right) \mathrm{m}^{2}$ \\
\hline Total Power & $\boldsymbol{O}\left(10^{3}\right) \div \boldsymbol{O}\left(10^{4}\right) \mathrm{W}$ & $\boldsymbol{O}\left(10^{2}\right) \mathrm{W}$ \\
\hline Dissipation Volume & $\boldsymbol{O}\left(10^{1}\right) \mathrm{dm}^{3}$ & $\boldsymbol{O}\left(10^{0}\right) \mathrm{dm}^{3}$ \\
\hline Volume Power Density & $\boldsymbol{O}\left(10^{2}\right) \div \boldsymbol{O}\left(10^{3}\right) \mathrm{W} / \mathrm{dm}^{3}$ & $\boldsymbol{O}\left(10^{2}\right) \mathrm{W} / \mathrm{dm}^{3}$ \\
\hline Cooling Loops & $\# \boldsymbol{O}\left(10^{1}\right)$ & $\# \boldsymbol{O}\left(10^{0}\right)$ \\
\hline
\end{tabular}

Table 1: Typical power figures in HEP Vertex detectors and modern computing chips

\section{Cooling solutions adopted at the LHC start}

For the first LHC run, very different solutions have been adopted for the different silicon detectors, often driven by considerations linked to the existing infrastructure. A dedicated Engineering Forum on cooling issues was held in 2008 [1], and interesting dedicated talks can be found in the 2011 Workshop on Quality issues [2:4]. In the two following sections only some generalities about the Vertex detectors of the four main experiments will be summarised.

\subsection{Off-detector cooling}

The use of water as refrigerant was discarded for the Vertex detectors at LHC mainly due 
to the risks of damage to the electronics in case of leaks. Furthermore, for the three detectors designed to operate below $0{ }^{\circ} \mathrm{C}$ (ATLAS Pixel, CMS PIX and LHCb Velo), the viscosity increase caused by the addition of freezing point depressive agents was considered excessive. On the other hand, the high level of radiation characteristic of the LHC operation prevents from circulating in a Vertex detector the refrigerating fluids most commonly in use in industrial applications. Indeed, under strong irradiation these fluids are not chemically stable and tend to polymerize and to recombine forming $\mathrm{HF}$ - with strong risks of pipe clogging and corrosion.

The class of perfluorocarbons $\mathrm{C}_{n} \mathrm{~F}_{2 \mathrm{n}+2}$ was finally chosen by ATLAS, CMS and ALICE, due to the chemical stability under irradiation and to the favourable dielectric properties of these molecules. However, rather different solutions were adopted for the cooling plants of the three detectors: $\mathrm{C}_{6} \mathrm{~F}_{14}$ in a 3-stage cold liquid pumped system for the CMS PIX [5, 6]; $\mathrm{C}_{3} \mathrm{~F}_{8}$ in a cold evaporative Joule-Thomson cycle for the ATLAS Pixel [6, 7]; $\mathrm{C}_{4} \mathrm{~F}_{10}$ in a room temperature evaporative Joule-Thomson cycle with an additional pump step for the ALICE SPD [8]. The main advantages of the 18-circuits, $3 \mathrm{~kW}$ liquid system selected by CMS are its simple design and the absence of complex regulation loops. However the long transfer lines through the experiment are cold (requiring a thorough insulation to avoid condensation risks) and larger in diameter than in an evaporative system. Also, the temperature rise of the liquid coolant along the pipes affects the uniformity of thermal conditions through the detector. The 88-circuits, $18 \mathrm{~kW}$ evaporative system of ATLAS, schematically represented in the pressure-enthalpy (p-h) diagram in Fig. 1(a), allows for reduced impact on the material budget, constant thermal conditions across the detector volume and warm transfer lines through the experiment. On the other hand, the system operation requires way more complex regulation loops, including a critical heating step in the detector volume; and it has been necessary to develop customized oilfree compressor units, out of existing industrial standards, requiring a continuous maintenance effort. The requirement of being lubricant-free for all active components potentially in contact with the detector pipework, again linked to the consequences of high radiation doses on possible oil contaminations in the circuits, actually makes extremely critical the design and operation of the compressor in a standard Joule-Thomson cycle. To mitigate this problem, taking advantage from the possibility of room temperature operation and reduced system temperature differential, the 10-circuits, $1.5 \mathrm{~kW}$ evaporative system developed for ALICE limits the compressor operation to a very low compression ratio and assign the larger pressure increase step to a liquid pump with rapid and inexpensive maintenance possibilities, placed in series to the condenser.
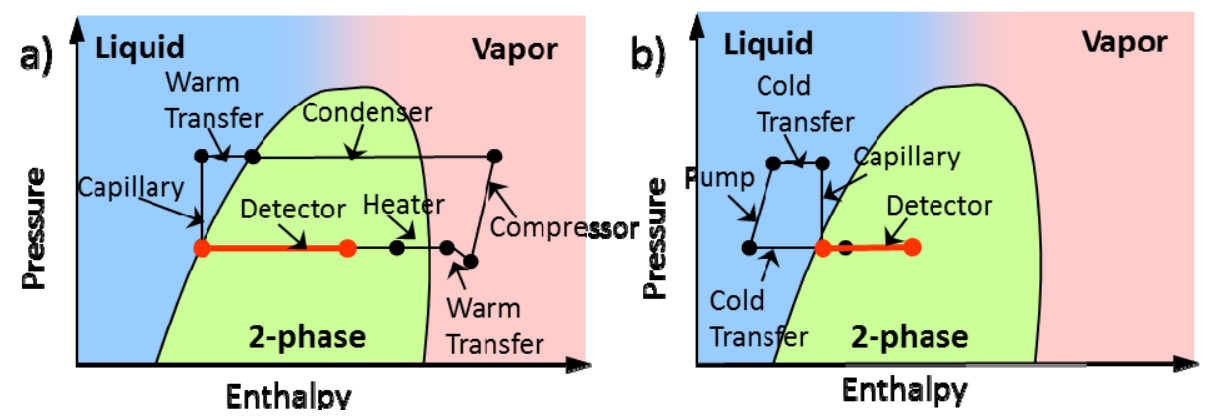

Figure 1: Comparison between the standard Joule-Thomson (a) and the 2PACL (b) cycle 
A completely different approach was adopted for the $1.5 \mathrm{~kW}$ cooling system of the LHCb Velo [9, 10]: a $\mathrm{CO}_{2}$ pumped evaporative loop controlled by a two-phase accumulator. The system, originally developed at NIKHEF with the name "2PACL" [11] for the AMS experiment at the International Space Station, combines the efficiency and the reduced mass of an evaporative cycle with the simplicity and reliability of a pumped liquid cycle (Fig.1 (b)). Liquid $\mathrm{CO}_{2}$ is cooled in a heat exchanger by a separated R507 industrial chiller (condensing unit) and pumped to the detector, where it expands in capillaries. The return fluid is then collected in a two-phase pressure vessel (the accumulator), which is the controlling element of the system: the combined action of a heater and a cooling spiral controls the pressure in the accumulator and therefore fixes the evaporating pressure (i.e. the temperature) in the detector loops. The main advantages of the 2PACL cycle are the absence of any active components in the detector loop, its operation stability and the very simple regulation required. Its main drawback is represented by the cold transfer lines through the experiment, which require adequate thermal insulation and humidity barrier. However, the extremely positive operation experience has made of it an important candidate for future detector upgrades, as will be discussed later.

\subsection{On detector cooling}

When designing the on-board cooling for a Vertex detector, all the particular requirements mentioned in the introduction must be addressed within a packed geometrical layout, only leaving limited space available for piping and connections. In order to define the local thermal management as "efficient", the following parameters must be minimized:

- The material budget $\mathrm{X} / \mathrm{X}_{0}$ of the planes crossed by particles;

- The temperature difference between heat source and heat sink;

- The temperature gradients on the surface of the sensor.

A rather traditional approach, very similar to those adopted for the silicon strip Trackers, has been followed for the four LHC Vertex detectors: a network of metallic pipes is added to a light-weight global support structure, optimized to provide a stable positioning to the detector modules with reduced material budget, and is brought in contact with the modules through some combination of heat spreaders, thermal contact materials and local heat sinks. The general scheme is represented in Fig.2. Aluminium, stainless steel and Phynox ${ }^{\circledR}$ were selected as pipe material (in addition, titanium and copper-nickel were also used for the strip Trackers) and the detailed engineering solution were very much configuration-driven. A very interesting review can be found in [12] along with a discussion of the parameters and issues considered.
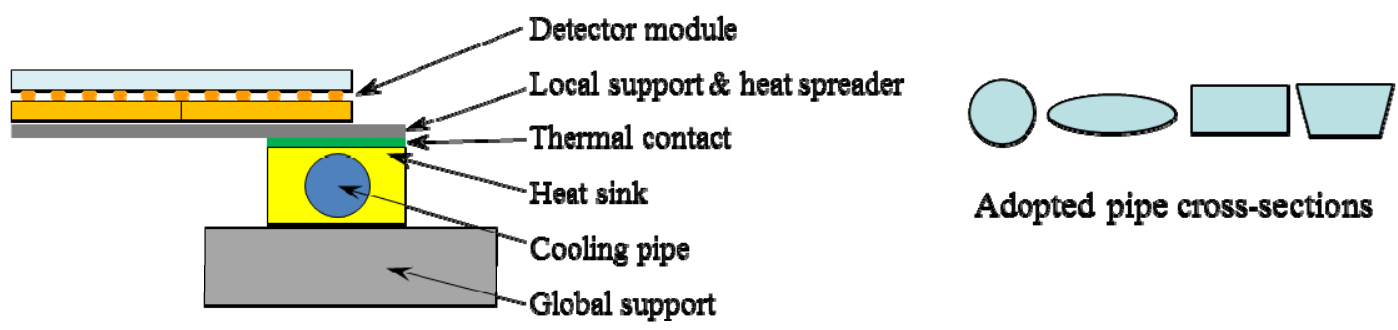

Adopted pipe cross-sections

Figure 2: General scheme of local thermal management for the four LHC Vertex detectors 
From a technical point of view, the main problem posed by the general scheme described is the mismatch of the Coefficient of Thermal Expansion (CTE) between the different materials in contact. Indeed, the relatively long thermal path between the local heat source and the cooling pipe requires minimizing each step of the chain of thermal resistances and therefore providing the best possible thermal contact at each step. However, intimate contact between materials with mismatched CTE may induce very high levels of stress, in particular in connection with the $40 \div 50{ }^{\circ} \mathrm{C}$ temperature step with respect to the assembly conditions, typical of the cold-operated detectors. Unless design options using materials with matching CTE or bringing the cooling fluid in direct contact with the module support are adopted, the resort to solutions requiring local compliant thermal joints is unavoidable. This, in turn, amplifies the issues of material budget, thermal resistance and long-term reliability. Nonetheless, this was the scheme adopted for all the present LHC Vertex detectors, mainly "because it leaves the maximum freedom in the design choices” [12].

\section{New trends for off-detector cooling systems}

The ambitious upgrade programme of LHC and its experiments implies even higher design goals for the future Vertex detectors, in terms of material budget, target temperature and reliability. As for the off-detector cooling systems, this translates in general design trends that can be summarized in the following points:

- General preference for evaporative systems;

- Preference to systems allowing for minimal pipe material in the detector volume;

- Suppression of oil-free compressors (at least with high compression ratios);

- Minimization of active components in the detector volume;

- Simplification of the control architecture.

\subsection{The Thermosiphon: a new concept for the ATLAS PIXEL and SCT detectors}

A radical approach toward the suppression of oil-free compressors and mechanical active components has been selected by ATLAS for the new cooling system of the ATLAS silicon Inner Detectors, including the Pixel: the so-called "thermosiphon system", conceived to substitute the critical compressor part of the present cycle. A thorough description of the system can be found in $[13,14]$, along with discussions of all the main technical aspects. The basic idea consists in condensing $\mathrm{C}_{3} \mathrm{~F}_{8}$ at the surface at very low temperature $\left(-65^{\circ} \mathrm{C}\right)$, and then exploiting the height difference between the surface and the ATLAS cavern to create a $95 \mathrm{~m}$ liquid column producing a 16 bars pressure at its bottom end. The high pressure, low temperature liquid is then heated in order to enter at a temperature of $+20^{\circ} \mathrm{C}$ the untouched existing detector loops, where it is expanded to evaporate at the requested temperature (nominal design: $-25^{\circ} \mathrm{C}$ ) in the detector volume. The natural circulation loop, without mechanical moving part in the main circuit, is closed by keeping the saturation pressure in the condenser, slightly lower than the evaporation pressure. The thermosiphon cycle combined with the existing detector cooling loop is schematically represented in the p-h diagram reported in Fig. 3. A three-step cascade system is connected to the condenser in surface: it is composed by a primary water system, a two-stage 
industrial chiller providing the required cooling power, and a $\mathrm{C}_{6} \mathrm{~F}_{14}$ pumped brine system to safely transfer the heat between the chiller and the condenser. The condenser is the central element of the system: the evaporation conditions inside the detector are indeed controlled through the regulation of its operation parameters. It is also capable of storing all the $2750 \mathrm{~kg}$ of $\mathrm{C}_{3} \mathrm{~F}_{8}$ contained in the system and of exchanging $170 \mathrm{~kW}$ of heat between the thermosiphon (main) circuit and the Brine circuit.

The control of the new ATLAS ID cooling system is composed of three main blocks: the Brine/Chiller system, the thermosiphon circuit and the Evaporative station. The latter is actually the previous ATLAS ID Evaporative Cooling Control System, already providing controls for the detector cooling loops, the evaporative heaters and the compressor-based plant and will continue to manage part of the cycle between point "I" and point "P" in Fig. 3. The whole control system is indeed designed to be fully compatible with the one operated until now: after completion of the commissioning, the thermosiphon will simply take the place of the present compressor stations with minimal disruption to the detector operation.

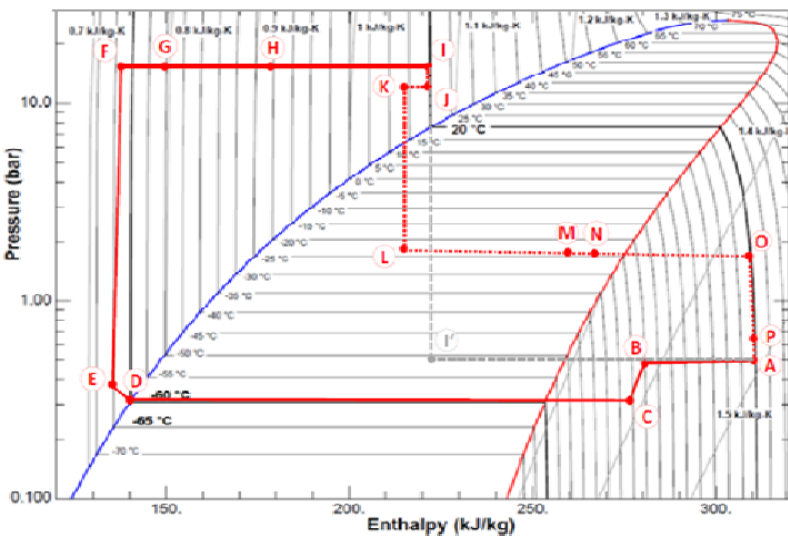

Figure 3: The new ATLAS Thermosiphon cycle (from [13])
Thermosiphon region:

A-B: Heat Exchanger (gas return line)

B-C: Return line pressure drop up to the surface

C-D: Condensation on surface

D-E: Subcooling (still in the condenser)

E-F: Compression along the 95 m liquid colnmm

F-G: First heater to tune the Heat Exchanger inlet temperature G-H: Heat Exchanger (liquid line in)

$\mathrm{H}-\mathrm{L}$ : Second Heater to bring the liquid to room temperature

Detector region (unteuched from present pIant)

I-J: Pressure regulator

J-K: On-detector Heat Exchanger (line in)

$\mathrm{K}-\mathrm{L}$ : Capillary expansion

L-M: DETECTOR

M-N: On-delecilor Heal Exchanger (relum line)

N-O: On-detector Heater to get $100 \%$ gas at room temperature

O-P: Back-pressure regulator

P-A: Relurn pressure crop up to the Heal Exchenger of $62 \mathrm{~kW}$ at $-25^{\circ} \mathrm{C}$ from the ATLAS ID volume requires a total electrical power of $233 \mathrm{~kW}$ ( $170 \mathrm{~kW}$ in surface to power the low temperature chiller and $63 \mathrm{~kW}$ for the two heating steps underground). After successful tests executed on two reduced-scale prototypes $[15,16]$, the final system is now under assembly and commissioning in ATLAS. Planned for immediate operation after the completion of the activities of the first LHC Long Shut down period (LS1), the thermosiphon system is intended to use $\mathrm{C}_{3} \mathrm{~F}_{8}$ as detector refrigerant. However, the system is also designed for possible circulation of a $\mathrm{C}_{3} \mathrm{~F}_{8} / \mathrm{C}_{2} \mathrm{~F}_{6}$ blend, for possible future lower temperature operation of the detector. In this case, it will be combined with a refrigerant mixer, controlled via precise real time monitoring of the return gas by an ad-hoc developed ultrasonic gas mixture analyser/flow meter [17]. 


\subsection{The CERN-NIKHEF standardized approach to $\mathrm{CO}_{2}$ cooling plants for HEP}

The interest of adopting $\mathrm{CO}_{2}$ as refrigerant for HEP applications has been well stressed in the last years $[18,19]$. For the same thermal performance (heat removed, temperature gradient), the unique combination of physical properties of $\mathrm{CO}_{2}$ allows for a sensitive reduction of the ondetector pipe diameter with respect to what achievable with standard synthetic refrigerants. This entrains a reduction in material budget and space consumption, clearly relevant to the design of future Vertex detectors. In addition, $\mathrm{CO}_{2}$ is inexpensive, has no ozone depleting properties, has the lowest greenhouse effect and bears no risk of phase-out from the market.

Leveraging on the success story and the proven reliability of the cooling systems of the AMS Silicon Tracker and the LHCb VELO, a programme has been launched, in collaboration between CERN PH-DT and NIKHEF, to develop a common approach to the design of 2PACL $\mathrm{CO}_{2}$ cooling plants. The first unit has been a $\mathrm{CO}_{2}$ test bench built at CERN in 2010 (CORA $\mathbf{C O}_{2}$ Research Apparatus) [20]. Offering cooling capacities up to $2 \mathrm{~kW}$ and open to external users, the system is conceived to test part of detectors or new components for future plants. The programme continuation aims to the definition of reliable standards for the design concept and the components, divided in three distinct classes of refrigeration units. Based on the reference standards, new units would then be customized in an interactive way taking into account the specific needs and constraints of the final client (e.g. HEP experiments or laboratories).

\subsubsection{The 15-20 kW class: CMS PIX plant prototype}

The largest unit of the proposed standard classes is a plant suited for direct application in HEP experiments for the thermal management of relatively large detectors. The first example of this class is the new plant for the Phase-I upgrade of the CMS PIX detector [21]. A prototype unit is presently completing it commissioning phase at CERN. With its $15 \mathrm{~kW}$ refrigeration power at $-20^{\circ} \mathrm{C}$ and its maximum mass flow rate of $150 \mathrm{~g} / \mathrm{s}$, this is the largest plant ever built using the 2PACL principle. After the definition of the standard logic and component set to be adopted for this class, the detailed dimensioning of the CMS specific unit has been performed in strict collaboration with the detector teams, in order to achieve an optimal combination of offdetector and on-detector parameters. This has largely contributed to the high reduction in material budget of the new CMS PIX detector. Based on the experience gained with the prototype construction, two identical units will be built and installed in CMS before the end of LS1. The two units will be organized in a redundant scheme, each one being able to cool down the full PIX detector if needed, but actually assigned only to half of it during normal operation.

At the present stage of development, we believe that this class of units could be the main building block for the $\mathrm{CO}_{2}$ cooling plants of future large upgrades: i.e. a parallel battery of units of this class could be used to serve very large silicon strip detectors, with power of the order of $100 \mathrm{~kW}$ and silicon surfaces in the order of $100 \mathrm{~m}^{2}$.

\subsubsection{The 1-2 kW class: ATLAS IBL and BELLE-II SVD/PXD plant prototype}

The mid-size class, typically delivering 1 to $2 \mathrm{~kW}$ of refrigeration power, covers the needs of small detectors or advanced testing in laboratory of complex portions of larger detectors. Implementing the full set of control, monitoring and interlock features designed for direct 
exploitation in a HEP experiment, this unit has dimensional characteristics $\left(1.3 \times 1.3 \times 2.4 \mathrm{~m}^{3}\right.$ volume for $300 \mathrm{~kg}$ weight) that also allows considering its shared use between different sites for laboratory tests. The prototype unit, MARCO (Multipurpose Apparatus for Research on CO2) [22] has been conceived as common prototype for the very similar requirements of the ATLAS IBL and the BELLE-II SVD/PXD detectors. In particular, the R-404a condensing unit of MARCO adopts a double stage compressor allowing for adequate sub-cooling $\left(\mathrm{T}<-50{ }^{\circ} \mathrm{C}\right)$ of the liquid $\mathrm{CO}_{2}$ at the pump, in order to deliver at $-40{ }^{\circ} \mathrm{C}$ the $20 \mathrm{~g} / \mathrm{s}$ maximum flow rate at the detector level. As the $-56{ }^{\circ} \mathrm{C}$ freezing point of $\mathrm{CO} 2$ is very close to the sub-cooling temperature, this requires extreme care for all the regulation elements and the full implementation of the controls of the main chiller in the PLC of the unit for finer control and faster reaction time.

MARCO has been the result of a fruitful collaboration between NIKHEF, CERN and MPI Munich. After its successful commissioning, it has served as baseline for the specific design of the final targeted detector cooling plants, presently under construction. The common prototype unit is now planned for long-term shared use between the laboratories.

\subsubsection{The 100-200 W class: a user-friendly unit for laboratory applications}

The last class of units developed, TRACI (Transportable Refrigeration Apparatus for $\mathbf{C O}_{2}$ Investigation) is the smallest in size and cooling power, conceived for non-expert use in routine small lab applications [23]. The design is based on a further simplification of the original 2PACL concept, named I-2PACL (for Integrated-2PACL), where all the liquid $\mathrm{CO}_{2}$ coming from the condenser is directly pumped in the accumulator and heated to the desired saturation temperature. This modification makes the cycle energetically less efficient, but allows for an easier control over a wide temperature range and reduces the pipework for construction. In consideration of the overall limited power targeted for this cooling unit (typically $600 \mathrm{~W}$ at room temperature and $100 \mathrm{~W}$ at $-30{ }^{\circ} \mathrm{C}$ ) its thermal inefficiency has been considered a negligible aspect. The modified concept is protected by a joint CERN-NIKHEF patent application and six prototypes have been fabricated until now. The prototypes are presently in use in different laboratories and, based on the operation and troubleshooting experience accumulated, a new version with optimised controls and hardware is presently being finalised. This latest version will also be intended for possible access offered to a wider community of end users. In parallel, the extension of the same concept to a more powerful (up to $1 \mathrm{~kW}$ at $-30^{\circ} \mathrm{C}$ ) unit is being pursued in collaboration with GSI [24].

\subsubsection{Control standardisation}

A fundamental aspect towards a standard approach to the design of $\mathrm{CO}_{2}$ cooling plants for HEP application is the definition of common philosophy for the design of the controls systems. An important effort has been made in this direction in terms of hardware, user interface, communication protocol and software development framework [25]. The common control architecture of all the systems developed relies on an industrial Ethernet IP field network and on highly reliable industrial hardware (e.g. Wago or Festo IP couplers, Siemens or Schneider PLCs). The user interface is based on the successful Siemens WinCC Open Architecture SCADA (Supervisory Control And Data Acquisition), and the control software conforms to the 
UNICOS CPC6 framework of CERN [26]. This approach simplifies the maintenance and operation activities both for the system experts and for the operators. Also, the use of state of the art industrial technology allows for a long lifetime perspective of the adopted standard.

An entirely new tool introduced in order to monitor the performance and to facilitate the commissioning of the plants features an on-line p-h diagram: the user interface panel is linked via dynamic libraries to the NIST Reference Fluid Thermodynamics and Transport Properties database (REFPROP) to retrieve on-line the coolant properties from current measurements. This produces in real time the representation of the thermodynamic cycle of the cooling unit in a p-h diagram on screen, enhancing the possibilities of rapid debugging and process analysis.

\section{Integrated design approach}

In any problem of conditioned optimum research for a function subject to multiple conditions, the global optimal solution is actually provided by the most effective weighted (i.e. prioritized) combination of sub-optimal single problem solutions. Likewise, when optimal performance is sought for a complex device that must comply with several conflicting requirements, it is conceptually wrong to expect that the optimal performance will be produced by the superposition of single optimal solutions to each constraint. The importance of an integrated engineering approach is therefore clearly emerging for the future generation of Vertex detector, for which one has to combine: very low temperature operation; increased total power; high stability in time; minimized mass and space consumption; increased radiation resistance; long-time reliability. This applies in particular to the thermal management issues, where the experience from the first LHC run already showed that the introduction a posteriori of additional elements, required to match and assemble highly optimized single components, might produce a global performance quite distant from expectations.

A wiser approach is to consider like an integral system the whole thermal path from the local on-detector heat sink, through the detector piping, to the long transfer lines up to the cooling unit, and to integrate this vision at the earliest possible stage of the detector conception.

\subsection{Detailed pipework engineering}

From the onset of boiling, when saturation conditions are reached, two-phase pipe flows develop into different flow regimes ("patterns") up to the appearance of mist flow at the end of the evaporation process (Fig. 4). Flow patterns have different thermal performances and are determined by the refrigerant mass flux and by its "vapour quality" (ratio between vapour and liquid), which depends in turn on the heat flux and saturation conditions. Optimal heat transfer is achieved when a flow rapidly moves to an "Annular" pattern, but never develops into mist, where "Dry-out" conditions appear at the pipe walls and the Heat Transfer Coefficient drops dramatically. By implementing state of the art correlations [27] into a computational model, it is possible to predict the pipe flow conditions for an assigned set of physical and geometrical parameters from the cooling station to the detector [28]. This allows for optimal balancing between transfer line geometry, manifolding and on-detector pipe dimensioning for any condition of the cooling plant. An example of calculation result is shown in Fig. 4, where the 


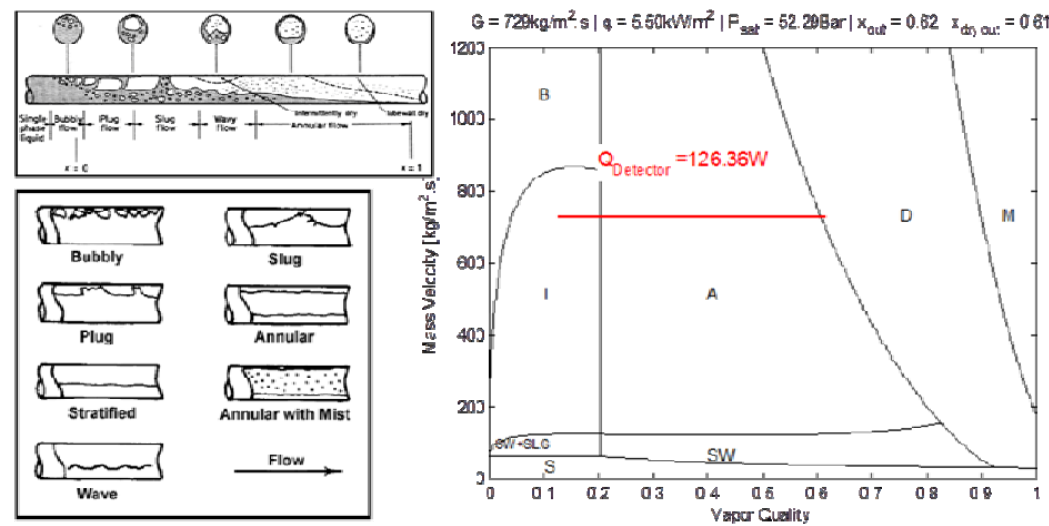

Figure 4: Different pipe flow patterns in evaporation and an example of flow map calculation for a $\mathrm{CO}_{2}$ pipe flow, based on $1 \mathrm{D}$ simulations and a flow pattern model

red line in the plot represents the calculated pattern in a pipe entering the detector very close to annular, but developing into early dry-out conditions before leaving the detector volume.

Correct dimensioning is only one aspect of optimal pipework design, which also requires early consideration of other fundamental issues. The first one is the adoption of reliable and well established standards for the selection of pipe materials, fittings and connection techniques [29]. The second is the provision for an adequate thermal insulation and humidity shielding of the cold pipe portions. In this particular respect, the adoption of specifically designed vacuum shielded pipes, a technique directly derived from cryogenics applications [30] has recently brought sensitive reduction of the total diameter of insulated pipes for the realization of the new cold transfer lines of the ATLAS IBL and CMS PIX detectors.

\subsection{On detector low-mass thermal management}

Under the push of the ever increasing demand for efficient electronics packaging, many highly performing materials are now available for the thermal management design of electronic devices. Carbon and graphite foams; highly conductive composites based on polymer, metal or ceramic matrices; new generation solderable Thermal Interface Materials and thermally conductive elastomers are examples of advanced materials proposed for industrial applications [31]. Due to the very specific constraint of HEP experiments, not all of them may find direct application in vertex detectors, but designers are looking at the possibilities offered by these developments with increasing interest [32]. The trend in this field is more and more towards a higher level of conceptual integration of the thermal management task into the lightweight support elements, in order to obtain low mass integrated cooling solutions [33]. This integral design approach has recently produced extremely interesting results, reported in Fig. 5. In the ATLAS IBL stave, a thin wall titanium pipe is embedded into highly conductive carbon foam, which is also the core of the sandwiched module support structure, producing a compact design with very low global thermal resistance [34]. The baseline stave proposed for the upgrade of the ALICE ITS detector combines polyimide pipes wrapped into layers of carbon fleeces and carbon paper with an ultra-lightweight carbon fibre space frame, achieving unparalled low mass 

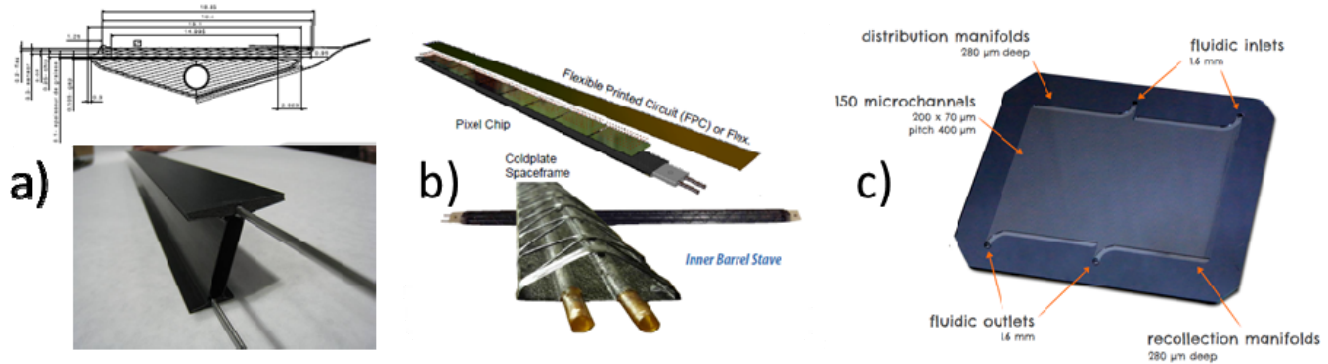

Figure 5: Examples of advanced low mass integrated thermal management solutions: ATLAS IBL stave (a), ALICE ITS stave (b) and NA62 GTK silicon micro-channel device

performance [35]. Micro-structured silicon devices, directly integrating the cooling channels with the detector modules for the lowest fluid-sensor $\Delta \mathrm{T}$ and no CTE mismatch, will be used for the first time in the GTK detector of NA62 at CERN, and are presently studied for the upgrade of the LHCb VELO, and also as an alternative solution for the future ALICE ITS [36].

The full exploitation of the benefits provided by these highly integrated low mass designs requires a well matched choice and control of all the refrigerant delivery parameters.

\section{Conclusions}

Several lessons about the relevance of a well-designed thermal management concept for future Vertex detectors have been retained from the experience of the first LHC run. Important efforts are on-going to bring innovative ideas into reality for the next generations of detectors. The main trends of development have been briefly reviewed in this paper. It is opinion of the author that optimal implementation of thermal management in future detectors cannot result from the superposition of solutions to separated issues, but from an integrated approach taking into account the whole thermal chain from the detector modules to the refrigeration plant.

\section{Acknowledgements}

The author is grateful to many colleagues for useful discussions and detailed information. In particular to M. Battistin, G. Bolla, C. Bortolin, J. Daguin, B. Di Girolamo, C. Gargiulo, D. Giugni, J. Godlewski, A. Mapelli, P. Tropea, B. Verlaat and L. Zwalinski.

Support for the development activities on $\mathrm{CO}_{2}$ plants has been received from the European Commission through the AIDA and CRISP collaborations in the frame of the FP7 programme.

\section{References}

[1] Engineering Forum: experiences from cooling systems for LHC detectors, CERN, Geneva, CH. 2008 https://indico.cern.ch/conferenceDisplay.py?confId=41288

[2] M. Battistin, Experience from Construction and Operation of the LHC Experiments Detectors Cooling Systems, in Workshop on Quality issues in current and future silicon detectors, CERN, Geneva, CH, 2011 https://indico.cern.ch/getFile.py/access?contribId=25\&sessionId=11\&resId=1\&materialId=slides\&c onfId $=148944$ 
[3] R. Turrisi, Flow rate issues in the ALICE SPD cooling, in Workshop on Quality issues in current and future silicon detectors, CERN, Geneva, CH, 2011

https://indico.cern.ch/getFile.py/access?contribId=28\&sessionId=11\&resId=2\&materialId=slides\&c onfId $=148944$

[4] B. Verlaat, Quality related experience in CO2 cooling systems, in Workshop on Quality issues in current and future silicon detectors, CERN, Geneva, CH, 2011 https://indico.cern.ch/getFile.py/access?contribId=26\&sessionId=11\&resId=0\&materialId=slides\&c onfId $=148944$

[5] P. Tropea, IT3230/TS/CMS - ADDENDUM to the Technical Specification for the cooling plant for the CMS Tracker and Preshower detectors, CERN EDMS doc 480997 (2004)

[6] K. Nagai, Cooling System Experiences from ATLAS and CMS, in proceedings of $18^{\text {th }}$ International Workshop on Vertex Detectors, Veluwe, NL, 2009, PoS (VERTEX 2009) 032

[7] D Attree et al, The evaporative cooling system for the ATLAS inner detector, JINST 3 (2008) P07003

[8] C. Bortolin, The ALICE experiment at CERN: development and commissioning of the cooling and control systems of the Silicon Pixel Detector and optimization of the LHC Exchange Interface project, CERN-THESIS-2010-262

[9] M. Van Beuzekom, A. Van Lysebetten, B. Verlaat, $\mathrm{CO}_{2}$ Cooling Experience (LHCb), in proceedings of $16^{\text {th }}$ International Workshop on Vertex Detectors, Lake Placid, NY, US, 2007, PoS (VERTEX 2007) 009

[10]B. Verlaat, A. Van Lysebetten, M. van Beuzekom, CO2 cooling for the LHCb-VELO experiment at CERN, in proceedings of the 8th IIF/IIR Gustav Lorentzen Conference on Natural Refrigerants, Copenhagen, DK, 2008, paper CDP 16-T3-08

[11]B. Verlaat, Controlling a Two-Phase $\mathrm{CO}_{2}$ Loop Using a Two-Phase Accumulator, in proceedings of the 2007 International Conference of Refrigeration, Beijing, CN, 2007, paper ICR07-B2-1565

[12]M. Olcese, Mechanics and cooling of pixel detectors, NIM A 465 (2001) p.51-59

[13]J.A. Botelho Direito, E. Perez Rodriguez, K. Egorov, L. Zwalinski, A. Bitadze, Thermosyphon Project Technical Note, CERN EDMS doc 1083852 (2010)

[14]J.A. Botelho Direito, ATLAS Thermosyphon Cooling Plant, CERN EDMS doc 1225594 (2012)

[15]J.A. Botelho Direito, E. Perez Rodriguez, Mini-Thermosyphon Tests Results, CERN EDMS doc 1083837 (2010)

[16]J.A. Botelho Direito, , PX15 Thermosyphon tests results, CERN EDMS doc 1245465 (2012)

[17]R. Bates et al., A combined ultrasonic flow meter and binary vapour mixture analyzer for the ATLAS silicon tracker, arXiv:1210.4835

[18]A.P. Colijn, B. Verlaat, CO2 Cooling Developments for HEP Detectors, in proceedings of $18^{\text {th }}$ International Workshop on Vertex Detectors, Veluwe, NL, 2009, PoS (VERTEX 2009) 031

[19]H. Postema, B. Verlaat, Cooling in HEP Vertex and Tracking Detectors, in proceedings of 20th International Workshop on Vertex Detectors, Rust, AT, 2011, PoS (VERTEX 2011) 003

[20]V.Bhanot, L.Zwalinski, J.Noite, H.Postema, J.Godlewski, T.Köttig, B.Verlaat, The CORA $\mathrm{CO}_{2}$ Cooling Plant, in proceedings of 10h IIF/IIR Gustav Lorentzen Conference on Natural Refrigerants, Delft, NL, 2012, paper GL-239 
[21]J. Daguin, K. Arndt, W. Bertl, J. Noite, P. Petagna, H. Postema, P. Tropea, B. Verlaat, Evaporative $\mathrm{CO}_{2}$ Cooling System for the Upgrade of the CMS Pixel Detector at CERN, in proceedings of $13^{\text {th }}$ IEEE ITHERM Conference, S. Diego, CA, US, 2012

[22]B. Verlaat, MARCO Conceptual Design Report, CERN EDMS doc 1345697 (2011)

[23]B. Verlaat, L. Zwalinski, R. Dumps, M. Ostrega, P. Petagna, T. Szwarc, TRACI, a Multipurpose $\mathrm{CO}_{2}$ Cooling System for $\mathrm{R} \& D$, in proceedings of $10 \mathrm{~h}$ IIF/IIR Gustav Lorentzen Conference on Natural Refrigerants, Delft, NL, 2012, paper GL-208

[24]J. Sanchez, J.M. Heuser, C.J. Schmidt, P. Petagna, B. Verlaat, L. Zwalinski, H. R. Schmidt, A. Lymanets, TRACI-XL, the Test Cooling System for the CBM Silicon Tracking System, CBM Progress Report 2012; ISBN 978-3-9815227-0-9, p. 21

[25]L. Zwalinski, J. Daguin, J. Godlewski, J. Noite, M. Ostrega, S. Pavis, P. Petagna, P. Tropea, B. Verlaat, The Control System for the $\mathrm{CO}_{2}$ Cooling Plants for Physics Experiments, in proceedings of 14th International Conference on Accelerator \& Large Experimental Physics Control Systems ICALEPCS 2013, S. Francisco, CA, US, paper MOPPC110 (to appear)

[26]Ph. Gayet, R. Barillère, UNICOS a Framework to Build Industry-like Control Systems Principles Methodo-logy, in proceedings of 10th International Conference on Accelerator \& Large Experimental Physics Control Systems ICALEPCS05, Geneva, CH, 2005, paper WE2.2-6I

[27]L. Cheng, G. Ribatski, J. Thome, New prediction methods for CO2 evaporation inside tubes: Part II- An updated general flow boiling heat transfer model based on flow patterns, Int. J. Heat and Mass Transfer, 51 (2008) p.111-124

[28]B. Verlaat, J. Noite, Design Considerations of Long Length Evaporative $\mathrm{CO}_{2}$ Cooling Lines, in proceedings of 10h IIF/IIR Gustav Lorentzen Conference on Natural Refrigerants, Delft, NL, 2012, paper GL-209

[29]R. French, Cooling: materials and joining technologies, in Workshop on Quality issues in current and future silicon detectors, CERN, Geneva, $\mathrm{CH}, 2011$

http://indico.cern.ch/getFile.py/access?contribId=27\&sessionId=11\&resId=1\&materialId=slides\&co $\underline{\text { nfId }=148944}$

[30]A. Kogan, J. Fesmire, W. Johnson, J. Minnick, Cryogenic vacuum thermal insulation systems, in proceedings of $23^{\text {rd }}$ International Cryogenic Engineering Conference, Wroclaw, PL, 2010

[31]X.C. Tong, Advanced Material for Thermal management of Electronic Packaging, Springer Editions, New York 2011

[32]B. Cooper, Low Mass Materials and Vertex Detector Systems, in this proceeding of $22^{\text {nd }}$ International Workshop on Vertex Detectors, Lake Starnberg, DE, 2013 (to appear)

[33]A. Mapelli et al., Low Mass Integrated Cooling, in this proceeding of $22^{\text {nd }}$ International Workshop on Vertex Detectors, Lake Starnberg, DE, 2013 (to appear)

[34]S. Coelli, M. Monti, IBL Stave Thermal Performance, CERN EDMS doc 1109283 (2010)

[35]The ALICE Collaboration, Upgrade of the ALICE Inner Tracking System, Technical Design Report CERN-LHCC-2013-024 (ALICE-TDR-017) (2013)

https://cds.cern.ch/record/1625842? ln=en

[36]A. Francescon, A. Mapelli, G. Nuessle, P. Petagna, A. Pezous, P. Renaud, G. Romagnoli, Application of Micro-channel Cooling to the Local Thermal Management of Detectors Electronics for Particle Physics, Microelectronics Journal, 44 (2013), p. 612-618 